\title{
Repercussões da infecção por Covid-19 em idosos: uma revisão integrativa
}

\author{
Repercussion of Covid-19 infection in the elderly: an integrative review \\ Repercusiones de la infección por Covid-19 en los ancianos: una revisión integrativa
}

Recebido: 25/11/2021 | Revisado: 02/12/2021 | Aceito: 15/01/2022 | Publicado: 16/01/2022

\author{
Nayara Alves Oliveira da Cruz \\ ORCID: https://orcid.org/0000-0001-8086-2249 \\ Faculdade de Ciências Médicas da Paraíba, Brasil \\ E-mail: nayaraalvesoliveiraa@gmail.com \\ Marcele Torres Andriani \\ ORCID: https://orcid.org/0000-0001-5005-2421 \\ Faculdade de Ciências Médicas da Paraíba, Brasil \\ E-mail: marceleandriani@gmail.com \\ Talita Saraiva Pimenta \\ ORCID: https://orcid.org/0000-0001-5753-7632 \\ Faculdade de Ciências Médicas da Paraíba, Brasil \\ E-mail: talitasaraiva202016@gmail.com \\ Antônio Ramos Nogueira Fernandes \\ ORCID: https://orcid.org/0000-0001-5209-3155 \\ Faculdade de Ciências Médicas da Paraíba, Brasil \\ E-mail: antonioramosnf@gmail.com \\ Rachel Cavalcanti Fonseca Pereira \\ ORCID: https://orcid.org/0000-0001-5457-397X \\ Faculdade de Ciências Médicas da Paraíba, Brasil \\ E-mail: rachel.fonseca@cienciasmedicas.com.br
}

\begin{abstract}
Resumo
O objetivo deste estudo foi realizar uma revisão integrativa da literatura a respeito das repercussões da infecção de Covid-19 em idosos. Para isso, utilizou-se artigos publicados entre os anos de 2020 a 2021 disponíveis nas seguintes bases de dados: ScienceDirect, Scielo, Lilacs, PubMED e Biblioteca Virtual de Saúde (BVS). A pesquisa foi realizada durante os meses de outubro e novembro. Selecionou-se artigos publicados em português e inglês, que resultou em 3.146 , destes foram incluídos apenas artigos que apresentassem no título a temática em estudo. Foram excluídos artigos duplicados e que não se enquadraram no objetivo da pesquisa. Após isso, elegeram-se 12 artigos para compor o universo da análise. Observa-se que o processo de envelhecimento natural inclui mudanças a nível celular, de tecidos e órgãos que contribuem para a morbidade, fragilidade e mortalidade na população idosa. Assim, os pacientes idosos infectados pelo Sars-Cov-2 possuem maior risco de desenvolver sintomas mais graves devido a redução do tamanho dos telômeros e ao número de linfócitos. Os estudos demonstraram que as repercussões mentais correlacionadas com o surto da Covid19 incluem o estresse, sintomas de depressão, ansiedade, insônia, indignação e o medo do desconhecido, incluindo risco aumentado de suicídio. Concluiu-se que a infecção por Covid-19 ainda é muito recente e algumas das suas repercussões e fisiopatologia ainda são desconhecidas. Os artigos selecionados não avaliaram as complicações após ao avanço da cobertura vacinal sobre esta população. Assim, sugere-se mais estudos voltados para a temática a partir deste ano de 2021.
\end{abstract}

Palavras-chave: Covid-19; Idoso; Repercussões pós infecção.

\begin{abstract}
The aim of this study was to carry out an integrative literature review on the repercussions of Covid-19 infection in the elderly. To achieve the objective, articles published between 2020 and 2021 were used and were available in the following databases: Sciencedirect, Scielo, Lilacs, Pubmed and Virtual Health Library (VHL). The articles were researched during the months of October and November. Articles published in Portuguese and English were selected. The search resulted in 3,146, but only articles that presented the theme under study were included in the title. Duplicate articles that did not fit the objective of the research were excluded. After the filtration process, 12 articles were chosen to compose the universe of Analysis. The natural aging process includes changes at the cellular level, tissues and organs that contribute to morbidity, frailty and mortality in the elderly population. Elderly patients infected with Sars-Cov-2 have a higher risk of developing more severe symptoms due to reduced telomere size and lymphocyte numbers. Studies have shown that mental disorders correlated with the Covid-19 outbreak include stress, symptoms of depression, anxiety, insomnia, indignation, and fear of the unknown, causing an increased risk of suicide. It was also concluded that the disease caused by Coronavirus is still very recent and some of its repercussions and physiopathology are still unknown. The selected articles did not evaluate the complications after the advancement of vaccine coverage in this population. Thus, we suggest more studies focused on the theme from this year of 2021.
\end{abstract}

Keywords: Covid-19; Elderly; Post infection repercussions. 


\begin{abstract}
Resumen
El objetivo de este estudio fue realizar una revisión integradora de la literatura sobre las repercusiones de la infección por Covid-19 en el anciano. Para alcanzar el objetivo se utilizaron artículos publicados entre los años 2020 a 2021 y que estuvieran disponibles en las siguientes bases de datos: ScienceDirect, Scielo, Lilacs, Pubmed y Biblioteca Virtual de Salud (BVS). Los artículos fueron investigados durante los meses de octubre y noviembre. Se seleccionaron artículos publicados en Portugués e inglés. La búsqueda resultó en 3.146, pero fueron incluidos solo artículos que presentaran en el título la temática en estudio. Fueron excluidos artículos duplicados y que no se encuadraron en el objetivo de la investigación. Después del proceso de filtración se eligieron 12 artículos para componer el universo de Análisis. El proceso de envejecimiento natural incluye cambios a nivel celular, de tejidos y órganos que contribuyen a la morbilidad, fragilidad y mortalidad en la población anciana. Los pacientes mayores infectados por el Sars-Cov-2 tienen mayor riesgo de desarrollar síntomas más graves, debido a la reducción del tamaño de los telómeros y al número de linfocitos. Los estudios han demostrado que los trastornos mentales correlacionados con el brote de Covid-19 incluyen el estrés, síntomas de depresión, ansiedad, insomnio, indignación y el miedo a lo desconocido, ocasionando un mayor riesgo de suicidio. Se concluyó además que la enfermedad ocasionada por el Coronavirus aún es muy reciente y algunas de sus repercusiones y fisiopatología aún son desconocidas. Los artículos seleccionados no evaluaron las complicaciones tras el avance de la cobertura vacunal sobre esta población. Así, se sugieren más estudios volcados para la temática a partir de este año 2021.
\end{abstract}

Palabras clave: Covid-19; Anciano; Repercusiones post infección.

\title{
1. Introdução
}

A Covid-19 é uma infeção causada pelo novo coronavírus, a cepa responsável por essa patologia foi identificada pela primeira vez em dezembro de 2019 em Wuhan, na China. Em janeiro de 2020 foi declarada uma emergência de saúde pública de importância internacional e em março desse mesmo ano foi declarada como pandemia pela Organização Mundial da Saúde (OMS) (Galvão \& Roncalio, 2020; Moraes et al., 2020; Nunes et al., 2020).

Desde então, a Covid-19 vem causando uma crise humanitária acarretando impactos sociais e econômicos no mundo inteiro. A transmissão da Covid-19 se dá, principalmente, por gotículas respiratórias, tosse, espirros, fluídos e também por superfícies de contato. Trata-se de um patógeno altamente contagioso e para que sua transmissibilidade seja reduzida, medidas sanitárias precisam ser tomadas, sendo fundamental para contenção do virus e redução ds mortes (Batista et al., 2020; Baptista \& Fernandes, 2020).

A clínica da doença varia desde assintomática à infeções graves, podendo ocasionar síndrome respiratória aguda grave, pneumonias complicadas, danos sistêmicos e até mesmo falência múltipla de órgãos. O prognóstico da Covid-19 torna-se pior na presença de fatores de risco. Os idosos são conhecidamente mais susceptíveis à forma grave da doença, pois apresentam múltiplos fatores de risco como diabetes mellitus, hipertensão arterial, doenças cardiovasculares e outras doenças de base que quando associadas, elevam ainda mais o risco de desenvolver formas severas e fatais da Covid-19 (Portela \& Cebola, 2021; Nunes et al., 2020).

A Covid-19 pode vir a causar déficit cognitivo com alterações de memória e fadiga mental é um dos principais sinais e sintomas dessa síndrome. Observa-se também, quadros de depressão e ansiedade, dores musculares e nas articulações, aumento da pressão arterial sem causa definida, entre outras queixas generalizadas (He et al., 2020). Diante deste cenário, objetivou-se com estudo realizar uma revisão integrativa da literatura a respeito das repercussões da infecção por Covid-19 em idosos.

\section{Metodologia}

$\mathrm{O}$ artigo trata-se de uma revisão integrativa da literatura, baseada na pesquisa bibliográfica de artigos científicos, com abordagem qualitativa, exploratória (Gerhardt \& Silveira, 2009). A busca dos artigos utilizadps para compor os resultados foi entre os meses de outubro e novembro de 2021. Os dados utilizados para desenvolvimento da pesquisa foram buscados nas bases de dados: ScienceDirect, Scielo. Lilacs, PubMED e Biblioteca Virtual de Saúde (BVS), optou-se por estes bancos de dados devido sua relevância no meio científico. As buscas foram realizadas a partir dos seguintes descritores em inglês: "Covid-19”, "Elderly", 
"Ageing" e em português: "Covid-19", "idoso" e "envelhecimento". Esses descritores foram interligados pelo operador booleano "and" para favorecer a busca dos estudos pelas bases de dados. Selecionou-se artigos nacionais e internacionais publicados entre os anos de 2020 e 2021 em periódicos e em Anais de congresso. Foi realizada uma análise prévia dos encontrados para posteriormente selecionar os que iriam compor os resultados com base nos critérios de inclusão.

A busca resultou em 4.205, porém foram incluídos apenas artigos que apresentasse no título e no resumo o tema proposto no objetivo de estudo, assim como artigos que estivessem disponiveis para download de forma gratuita. Foram excluídos artigos duplicados e que não se enquadraram no objetivo da pesquisa. Após o processo de filtragem e leitura dos textos na íntegra, elegeram-se 6 artigos para compor o universo de análise que foram consideradas os mais relevantes para a obtenção do objetivo proposto conforme Figura 1.

Figura 1. Artigos selecionados segundo as bases de dados.
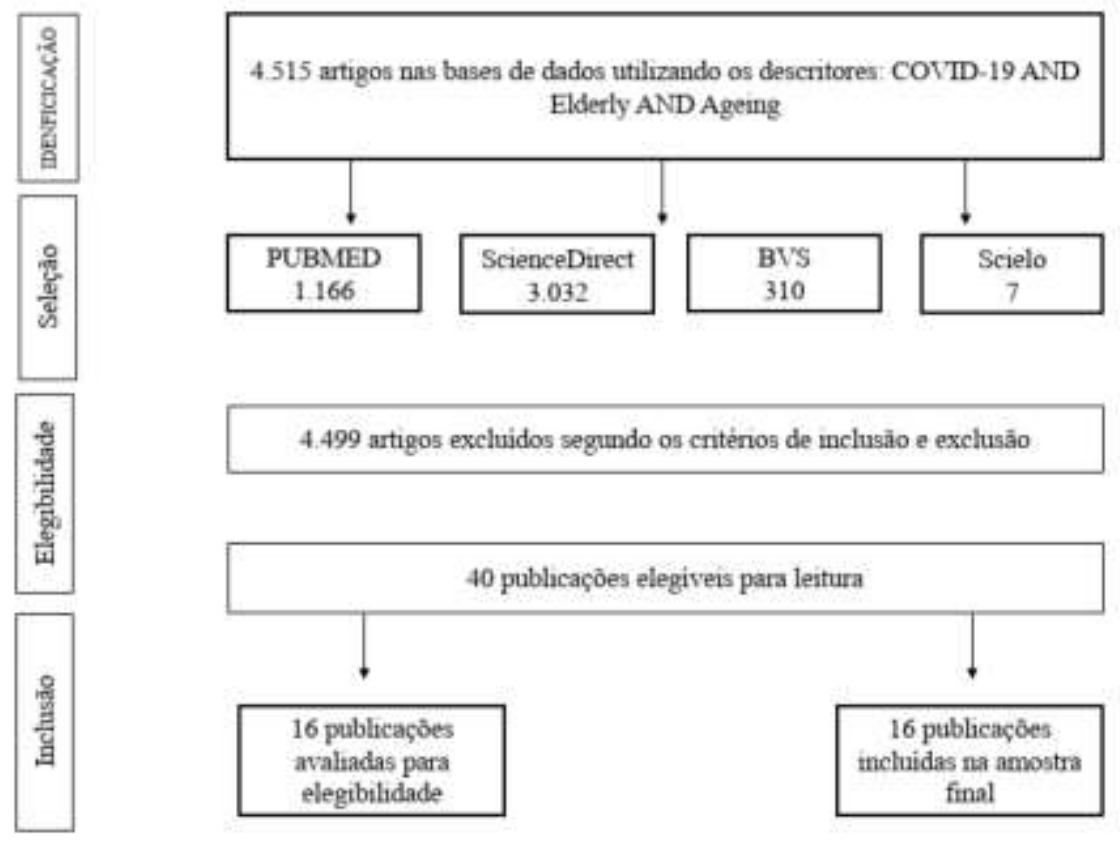

Fonte: Autores.

Para desenvolvimento dos resultados utilizou-se o Sistema GRADE (Grading of Recommendatons Assessment, Development and Evaluaton), onde pode-se apresentar evidência procedente de ensaios clínicos com base no nivel de qualidade do artigo em estudo. Os resultados foram apresentados sob a forma de tabela. As variáveis para elaboração dos resultados foram: autor, ano de publicação, título, objetivos e principais resultados. Estes itens foram considerados suficientes para descrever os resultados de maneira sucinta e relevante ao objeto de estudo. Após a análise da tabela, os dados foram agrupados em três categorias para posterior discussão, sendo elas: repercussões da infecção por Covid-19 no envelhecimento; mecanismo clínico e implicações imunológicas do Covid-19; complicações orgânicas pós infecção pela Covid-19 em indivíduos idosos; e repercussões psicológicas por Covid-19 nos idosos.

\section{Resultados e Discussão}

O presente estudo contou com 12 artigos que foram distribuídos conforme a Tabela 1. Os artigos referem o mecanismo clínico da Covid-19, bem como a resposta imunológica do indivíduo à infecção. 
Tabela 1. Sistematização do corpus de análise da pesquisa acerca das repercussões após infecção por Covid em Idosos: uma revisão integrativa.

\begin{tabular}{|c|c|c|c|c|c|}
\hline $\begin{array}{c}\text { Periódico e ano de } \\
\text { publicação }\end{array}$ & Autores & Título do artigo & Objetivo do estudo & Metodologia & Resultados \\
\hline $\begin{array}{c}\text { International Journal } \\
\text { of } \\
\text { Molecular Sciences, } \\
2021 .\end{array}$ & $\begin{array}{c}\text { Mongelli et } \\
\text { al. }\end{array}$ & $\begin{array}{l}\text { Evidence for Biological } \\
\text { Age Acceleration and } \\
\text { Telomere shortening in } \\
\text { COVID-19 Survivors. }\end{array}$ & $\begin{array}{c}\text { Observar entre os } \\
\text { sobreviventes da covid a } \\
\text { presença da síndrome } \\
\text { persistente pós-Covid-19 } \\
\text { (PPCS) e o encurtamento } \\
\text { dos telômeros em } \\
\text { comparação a pessoas não } \\
\text { infectadas. } \\
\end{array}$ & $\begin{array}{l}\text { Estudo de } \\
\text { coorte. }\end{array}$ & $\begin{array}{l}\text { Foi demonstrado que indivíduos } \\
\text { pertencentes a um grupo de } \\
\text { sobreviventes da COVID-19 exibiram } \\
\text { uma significativa aceleração de sua } \\
\text { idade biológica, estando relacionada } \\
\text { ao encurtamento dos telômeros, } \\
\text { favorecendo ao aparecimento da } \\
\text { Síndrome Persistente Pós Covid-19. }\end{array}$ \\
\hline $\begin{array}{l}\text { Journal of nutrition, } \\
\text { health \& aging, } \\
2020 .\end{array}$ & $\begin{array}{c}\text { Saban-Ruiz \& } \\
\text { Ly-Pen. }\end{array}$ & $\begin{array}{c}\text { Covid-19: A } \\
\text { Personalized } \\
\text { Cardiometabolic }\end{array}$ & $\begin{array}{l}\text { Analisar os benefícios da } \\
\text { abordagem } \\
\text { cardiometabólica na } \\
\text { prevencão de complicacões }\end{array}$ & $\begin{array}{l}\text { Estudo de } \\
\text { Revisão de } \\
\text { Literatura. }\end{array}$ & $\begin{array}{l}\text { Foram encontradas várias } \\
\text { complicações cardiovasculares que } \\
\text { surgem durante a hospitalização. }\end{array}$ \\
\hline
\end{tabular}

Approach for Reducing prevenção de complicações

Complications And do Covid-19

Costs.the Role Of Aging

\begin{tabular}{|c|c|c|c|}
\hline Journal of the Royal & Robinson \& & Is 'inflammaging' & Analisar o espectro de \\
\hline $\begin{array}{c}\text { Society of Medicine, } \\
2020 .\end{array}$ & Pierce. & $\begin{array}{c}\text { fuelling severe COVID- } \\
19 \text { disease? }\end{array}$ & $\begin{array}{l}\text { gravidade da doença com a } \\
\text { infecção por COVID-19 }\end{array}$ \\
\hline
\end{tabular}

\begin{tabular}{|c|c|c|c|c|}
\hline $\begin{array}{c}\text { International } \\
\text { Archives of } \\
\text { Otorhinolaryngolog } \\
\mathrm{y}, 2020 .\end{array}$ & $\begin{array}{l}\text { Begam \& } \\
\text { Basha. }\end{array}$ & $\begin{array}{c}\text { Olfactory and Taste } \\
\text { Disorders in Patients } \\
\text { with SARS-CoV-2 } \\
\text { Infection. }\end{array}$ & $\begin{array}{l}\text { Observar a presença de } \\
\text { alterações olfativas e } \\
\text { gustativas em pacientes } \\
\text { com Covid-19. }\end{array}$ & $\begin{array}{c}\text { Estudo } \\
\text { Transversal. }\end{array}$ \\
\hline $\begin{array}{l}\text { Neuroepidemiology, } \\
2020 .\end{array}$ & $\begin{array}{l}\text { Beghi, Feigin, } \\
\text { Caso, } \\
\text { Santalucia \& } \\
\text { Logroscino. }\end{array}$ & $\begin{array}{c}\text { COVID-19 Infection and } \\
\text { Neurological } \\
\text { complications: Present } \\
\text { Findings and future } \\
\text { Predictions. }\end{array}$ & $\begin{array}{c}\text { Analisar as complicações } \\
\text { neurológicas em pacientes } \\
\text { portadores da Covid-19 }\end{array}$ & Metanálise. \\
\hline
\end{tabular}

Predictions.

\begin{tabular}{ccccc}
\hline The Journals of & Salimi \& & COVID-19 and & Observar as características & Estudo de \\
Gerontology: Series & Haml. & Crosstalk with the & do envelhecimento com a \\
A, 2020. & & Hallmarks of Aging. & infecção da Covid-19 & Revisão.
\end{tabular}
A suscetibilidade diferencial que faz
com que alguns indivíduos progridam
para doenças graves está associada a
um sistema imunológico hiperativo
ativado com inflamação crônica.

Alterações olfativas e gustativas são bastante frequentes em pacientes com infecção por SARS-CoV-2 e pode preceder o início da doença clínica desenvolvida.

O estudo apresenta limitações, pois as informações disponíveis sobre a frequência de distúrbios neurológicos em pessoas com Covid-19 são baseadas em séries clínicas, que fornecem apenas um quadro aproximado e talvez tendencioso da associação entre a doença e complicações neurológicas.

Tanto o processo de imunosenescência inata como adaptativa influenciam na gravidade da COVID-19. Após a infecção pelo vírus é ativado alguns receptores de defesa que estimulam citocinas próinflamatórias e interferons (IFNs). O coronavírus pode antagonizar os IFNs e, assim, invadir o sistema imunológico.

\begin{tabular}{ccc}
\hline $\begin{array}{c}\text { Journal of the } \\
\text { American Medical } \\
\text { Directors }\end{array}$ & Tosato et al. & $\begin{array}{c}\text { Prevalence and } \\
\text { predictors of persistence } \\
\text { of COVID-19 symptoms } \\
\text { in older adults: a single- } \\
\text { center study. }\end{array}$ \\
\hline $\begin{array}{c}\text { Cadernos de Saúde } \\
\text { Pública, 2021 }\end{array}$ & Romero et al. & $\begin{array}{c}\text { Older adults in the } \\
\text { context of the COVID- } \\
19 \text { pandemic in Brazil: } \\
\text { effects on health, income } \\
\text { and work. }\end{array}$ \\
& & $\begin{array}{c}\text { and } \\
\end{array}$
\end{tabular}

$\begin{array}{cc}\text { Determinar a prevalência } & \text { Estudo } \\ \text { de sintomas persistentes } & \text { Transversal. } \\ \text { entre os idosos } & \end{array}$

Dos pacientes que se recuperaram da COVID-19, mais de $80 \%$ reportaram persistência de ao menos um sintoma após a infecção, sendo os principais relatados: fadiga, dispneia, tosse e dor sobreviventes ao COVID19 e identificar padrão de sintomas. nas articulações.

Caracterizar a população

de idosos no Brasil durante Estudo O estudo evidencia que sentimentos de a pandemia COVID-19 quanto à saúde, condições

Transversal solidão, angústia e tristeza foram frequentes entre os idosos, principalmente entre as mulheres. Além disso, a pandemia pelo Covid19 ampliou a lacuna de desigualdade ao afetar os idosos mais vulneráveis.

desigualdade de gênero, adesão e distanciamento social e sentimentos de tristeza ou depressão.

$\begin{array}{ccccc}\text { Anais do Congresso } & \text { Costa et al. } & \text { Impacto do COVID-19 } & \text { Promover a visibilidade } & \text { Revisão } \\ \text { Internacional de } & \text { na qualidade de vida dos } & \text { sobre o impacto na saúde } & \text { sistemática da } \\ \text { Envelhecimento } & & \text { idosos. } & \text { causada pelo COVID-19 } & \text { literatura. } \\ \text { Humano, 2020. } & & \text { em idosos. } & \end{array}$

Relata que o isolamento, a redução da atividade física e a diminuição das visitas e o estresse durante o período de pandemia impactaram na qualidade de vida dos idosos. Além disso, surgiram sentimentos de incerteza, medo e angústia na população idosa, ao passo que emergiram os sentimentos de responsabilidade e cuidado mediante a pandemia. Além disso, evidencia que o impacto na 


\begin{tabular}{|c|c|c|c|c|c|}
\hline & & & & & $\begin{array}{l}\text { saúde mental é desigual de acordo } \\
\text { com o gênero, nível educacional e } \\
\text { ambiente familiar. }\end{array}$ \\
\hline $\begin{array}{l}\text { Brazilian Journal of } \\
\text { Development, } 2020 .\end{array}$ & Costa et al. & $\begin{array}{l}\text { COVID-19 e seus } \\
\text { impactos clínicos e } \\
\text { psicológicos na } \\
\text { população idosa. }\end{array}$ & $\begin{array}{c}\text { Analisar e investigar } \\
\text { através de uma revisão da } \\
\text { literatura os impactos do } \\
\text { novo coronavírus no } \\
\text { metabolismo e psicológico } \\
\text { dos idosos e possíveis } \\
\text { intervenções que possam } \\
\text { minimizar esses impactos. }\end{array}$ & $\begin{array}{c}\text { Revisão } \\
\text { qualitativa } \\
\text { exploratória } \\
\text { da literatura. }\end{array}$ & $\begin{array}{l}\text { O estudo evidencia que o sentimento } \\
\text { de medo em ser acometido pelo } \\
\text { SARS-Cov-2 devido aos sintomas } \\
\text { associados à infecção. Esse } \\
\text { sentimento interfere nas atividades de } \\
\text { vida diária e por conseguinte, no } \\
\text { psicológico dos idosos e suas funções } \\
\text { corporais. }\end{array}$ \\
\hline $\begin{array}{c}\text { Journal of the } \\
\text { American Medical } \\
\text { Directors } \\
\text { Association, } 2021 .\end{array}$ & Briggs et al. & $\begin{array}{l}\text { Depressive symptoms } \\
\text { among older adults pre } \\
\text { and post COVID-19 } \\
\text { pandemic. }\end{array}$ & $\begin{array}{l}\text { Examinar a tendência de } \\
\text { sintomas depressivos e } \\
\text { determinar a prevalência } \\
\text { desses sintomas antes e } \\
\text { depois da pandemia por } \\
\text { COVID-19. }\end{array}$ & $\begin{array}{c}\text { Estudo } \\
\text { longitudinal. }\end{array}$ & $\begin{array}{l}\text { O estudo evidencia que os sintomas } \\
\text { depressivos aumentaram no período } \\
\text { da pandemia pelo COVID-19, } \\
\text { principalmente nos idosos com mais } \\
\text { de } 70 \text { anos e/ou que moram sozinhos. }\end{array}$ \\
\hline $\begin{array}{c}\text { Saudi Journal of } \\
\text { Biological Sciences, } \\
2021\end{array}$ & $\begin{array}{l}\text { Sharma \& } \\
\text { Sharma. }\end{array}$ & $\begin{array}{l}\text { Impact of COVID-19 on } \\
\text { mental health and aging. }\end{array}$ & $\begin{array}{c}\text { Investigar fatores } \\
\text { associados à pandemia que } \\
\text { possuem efeitos } \\
\text { prejudiciais à saúde mental } \\
\text { e sua interferência no } \\
\text { envelhecimento. }\end{array}$ & $\begin{array}{l}\text { Estudo de } \\
\text { revisão. }\end{array}$ & $\begin{array}{l}\text { A pandemia pelo COVID-19 possui } \\
\text { um efeito prejudicial sobre a saúde } \\
\text { psicológica e o envelhecimento. A } \\
\text { mudança no estilo de vida, o } \\
\text { isolamento e a quarentena, bem como } \\
\text { o distanciamento social e o transtorno } \\
\text { de estresse pós-traumático são } \\
\text { evidentes. }\end{array}$ \\
\hline
\end{tabular}

Fonte: Autores.

Dentre os principais achados, pode-se destacar a relação do envelhecimento com a infecção pelo coronavírus, que parece estar relacionado com quadros clínicos mais graves e complicações pós Covid-19. Além disso, pode-se destacar as complicações orgânicas encontradas nos estudos, sendo a principal a insuficiência respiratória. A complicação cardiometabolica também é descrita, estando relacionada principalmente às comorbidades: diabetes melitus e hipertensão. Outras complicações citadas são as alterações gustativas e olfativas, além do comprometimento neurológico e a síndrome persistente pós Covid-19. Por fim, os estudos apontam as consequências psicológicas relacionadas ao coronavírus e ao isolamento social, sendo os principais achados o estresse, a depressão e o medo.

De acordo com a presente revisão sistemática, as áreas temáticas observadas foram categorizadas em: repercussões da infecção por Covid-19 no envelhecimento; mecanismo clínico e implicações imunológicas do Covid-19; complicações orgânicas pós infecção pela Covid-19 em indivíduos idosos; e repercussões psicológicas por Covid-19 nos idosos.

\section{Repercussões da infecção por Covid-19 no envelhecimento}

O processo de envelhecimento natural inclui mudanças a nível celular, de tecidos e órgãos que contribuem para a morbidade, fragilidade e mortalidade na população idosa. As características do envelhecimento vão interferir em todos os aspectos das funções celulares e do sistema orgânico ao interagir direta ou indiretamente com uma infecção viral. Algumas dessas características incluem imunosenescência, processo de inflamação, imunosenescência adaptativa, instabilidade genômica, disfunção mitocondrial, alterações epigenéticas, disfunção dos telômeros e autofagia prejudicada que resulta na capacidade de adaptação em resposta ao estresse (Salimi \& Hamlyn, 2020).

A imunosenescência se refere ao processo inflamatório crônico que ocorre em doenças relacionadas a idade associadas ao declínio na eficácia do sistema imunológico, onde as citocinas pró-inflamatórias estão significativamente elevadas, especialmente a citocina Interleucina 6, que é uma interleucina liberada pelo Coronavírus que ocasiona complicações graves pulmonares como a Síndrome da Angústia Respiratória Aguda (SDRA) (Robinson \& Pierce, 2020).

Pesquisas apontam que o encurtamento dos telômeros está associado ao processo de envelhecimento devido a senescência celular replicativa. Os telômeros possuem propriedades que os tornam biomarcadores em várias doenças ou condições, incluindo neoplasias e doenças cardiovasculares. A correlação inversa entre o comprimento dos telômeros e a idade cronológica é um fator que tem sido usado para a previsão da idade. Estima-se que indivíduos mais velhos infectados pelo Sars- 
Cov-2 tenham um risco maior de desenvolver sintomas mais graves devido a redução do tamanho dos telômeros e ao número de linfócitos (Mongelli, 2021).

\section{Mecanismo clínico e implicações imunológicas da Covid-19}

O Coronavírus $(\mathrm{CoV})$ é vírus com RNA de fita simples positivo pertencente à família coronaviridae da ordem dos Nidovirales, que são responsáveis por doenças em animais (ACoV), mas também pode causar doenças em humanos (HCoV). É um vírus antigo que já causou doenças em seres humanos que variam desde o resfriado comum até doenças mais graves, como SARS e Síndrome Respiratória do Oriente Médio (MERS) (Saban-Ruiz \& Ly-Pen, 2020). A Covid-19 tem essa nomenclatura devido ao foco inicial ter se desenvolvido em Wuhan (Hubei, China) no período de dezembro de 2019. Em 11 de fevereiro de 2020, a Organização Mundial de Saúde (OMS) reconheceu esta nova doença e denominou o novo vírus como SARS-CoV2 (Saban-Ruiz \& Ly-Pen, 2020).

A Covid-19 apresenta alta capacidade de virulência e rápida transmissibilidade, principalmente por meio de gotículas de aerossol, fator com que fez com que a doença se espalhasse rapidamente pelo mundo. Se manifesta tanto de forma assintomática quanto de forma sintomática, se apresentando com ou sem febre, tosse, falta de ar, fadiga e sintomas gastrointestinais. Pode evoluir para uma pneumonia moderada ou grave, síndrome do desconforto respiratório agudo sintomático grave, complicações cardiovasculares, lesão renal, acidente vascular cerebral, gerando morbidade e mortalidade. Nos exames laboratoriais, a maioria dos pacientes apresentam uma "tempestade de citocinas", caracterizadas por leucopenia, trombocitopenia e coagulopatia. A tomografia computadorizada apresenta opacidades em vidro fosco multifocais e áreas subsegmentares de consolidação e fibrose (Salimi \& Hamlyn, 2020).

Sabe-se que o coronavírus além do envolvimento do sistema respiratório atinge o sistema nervoso, podendo ser decorrente de uma ação direta desse vírus no tecido nervoso e ou de uma ação indireta por meio da ativação de mecanismos imunomediados. Enquanto a primeira ação pode ser verificada durante a fase aguda da doença, a segunda pode ser observada apenas após dias, semanas ou meses após a fase aguda. O coronavírus invade os tecidos nervosos envolvendo macrófagos com função imunológica, microglia ou astrócitos e causam danos aos nervos por meio de vias de infecção diretas (circulatórias e neuronais), hipóxia e lesão imunológica (Beghi et al., 2020).

\section{Complicações orgânicas pós infecção pela Covid-19 em indivíduos idosos}

As doenças crônicas tem um vínculo significativo com a evolução clínica da Covid-19 podendo gerar complicações, uma vez que a combinação de diferentes problemas de saúde causa um processo inflamatório, aumentando, assim, a sensibilidade do paciente as infecções e ocasionando a redução da sua imunidade (Costa, 2020). A marca registrada da doença grave é a insuficiência respiratória com áreas de pneumonia. Os pacientes relatam que sentem que estão se afogando e progridem rapidamente para SDRA. Este quadro clínico resulta do vírus desencadear uma intensa inflamação, com danos às paredes dos alvéolos, havendo uma liberação descontrolada de citocinas pró-inflamatórias, especialmente a Interleucina - 6, que irá danificar células pulmonares (Robinson \& Pierce, 2020).

Já se tinha o conhecimento das complicações respiratórias, hoje surge uma faceta menos conhecida que é a cardiometabólica, que pode preceder, acompanhar ou mesmo causar o óbito se não for observada. Dentro dos aspectos cardiometabólicos da Covid-19, leva-se em consideração o Diabetes Mellitus e a hipertensão, os quais estão estritamente relacionados à idade, ganho de peso e baixa atividade física. A presença dessas duas comorbidades podem agravar o quadro da doença podendo gerar uma síndrome metabólica e aumento do risco cardiovascular (Saban-Ruiz \& Ly-Pen, 2020).

Muitos pacientes que apresentam infecção por Covid-19 relatam a presença de distúrbios olfativos e gustativos. Em um estudo transversal da prevalência dessas alterações no contexto da SARS-CoV-2 em pacientes internados por COVID-19 no 
Hospital de Doenças Infecciosas em Milão, na Itália, 59 foram selecionados, destes apenas 20 (33,9\%) pacientes tinham condições de responder a pesquisa e relataram pelo menos 1 distúrbio gustativo ou olfativo e 11(18,6\%) relataram ambos. Doze pacientes $(20,3 \%)$ apresentaram os sintomas antes da internação, enquanto $8(13,5 \%)$ experimentaram os sintomas durante a hospitalização, então conclui-se que as alterações gustativas e olfativas são mais presentes no início da doença (Begam \& Bashar, 2020).

Um outro aspecto observado nesse estudo, envolve o acometimento do sistema nervoso pelo coronavírus. Achados neurológicos durante o surto de COVID-19 foram descritos em um estudo em Wuhan, China. Manifestações neurológicas estavam presentes em 78/214 pacientes (36,4\%) e foram divididas em 3 categorias: sistema nervoso central (AVC, tontura, dor de cabeça, consciência prejudicada, ataxia e convulsões), sistema nervoso periférico (comprometimento do paladar, comprometimento do olfato, comprometimento da visão e dor neural) e músculo-esquelético (ataxia, convulsões e depressão do nível de consciência). Os pacientes com complicações mais graves eram idosos e apresentavam comorbidades médicas (Beghi, 2020).

E ainda, foi encontrado em alguns pacientes sintomas caracterizados como a Síndrome Persistente Pós Covid-19. Segundo a Organização Mundial da Saúde (OMS), o tempo de recuperação da doença é de aproximadamente 2 semanas nos casos leves e de 3 a 6 semanas nas infecções mais graves. No entanto, uma parcela considerável de pacientes se queixa de sintomas relacionados a Covid-19 semanas após o início da doença. Os sinais e sintomas comuns de longa duração incluem tosse, febre, dispneia, fadiga, queixas musculoesqueléticas (mialgia, dores nas articulações), gastrointestinais, anosmia e disgeusia (Tosato, 2021).

\section{Repercussões psicológicas por Covid-19 nos idosos}

A pandemia trouxe diversas repercussões nas vidas das pessoas, incluindo a ameaça a vida, perda de entes queridos, maior risco de pobreza, perda de suporte social, trauma de estigma, discriminação e isolamento. Esses fatores irão influenciar nos aspectos psicológicos vivenciados durante a Covid-19. A perda do rendimento familiar durante a pandemia poderá agravar as desigualdades sociais e de saúde, causando grande sentimento de tristeza e afetando a esperança de uma vida saudável (Romero et al, 2021).

Vale ressaltar que uma boa saúde mental é tão importante quanto o cuidado com a saúde física. Estudos apontam que os transtornos mentais correlacionados com a Pandemia da Covid-19 incluem o estresse, sintomas de depressão, ansiedade, insônia, indignação e o medo do desconhecido, ocasionando um risco aumentado ao suicídio. Em um estudo realizado na China foi registrado que 16,5\% dos indivíduos com Covid-19 apresentaram sintomas médios a graves de depressão, 28,8\% de sintomas médios a graves de ansiedade e $8,1 \%$ de níveis moderados a graves de estresse. Portanto, há uma alta prevalência de problemas de saúde mental correlacionados com a exposição regular as redes sociais durante a pandemia (Sharma \& Sharma, 2021).

O isolamento social entre os idosos constitui uma séria preocupação de saúde pública devido ao risco aumentado de comorbidades como doenças cardiovasculares, autoimunes, distúrbios neurocognitivos e de saúde mental. Idosos que apresentam doenças mentais, são mais propensos a depressão e ansiedade, e correm um risco muito maior de recaída. Tendo em vista que muitos idosos são dependentes de outras pessoas para a prática de atividades de vida diárias, o distanciamento social os fez sentirem sozinhos, aumentando o risco de efeitos adversos na saúde mental (Costa, 2020).

Em uma pesquisa descritiva realizada pela Fundação Oswaldo Cruz no Brasil o sentimento de solidão devido ao distanciamento social durante a pandemia foi relatado por metade dos idosos, sendo este sentimento mais frequente no sexo feminino $(57,8 \%)$, em relação ao masculino (41\%). Foram relatados ansiedade ou nervosismo em 1/3 da população idosa $(31,7 \%)$, sendo maior também entre as mulheres $(38,1 \%)$, do que entre os homens $(23,2 \%)$. O sentimento recorrente de tristeza e depressão (27,5\%) também foi mais marcante na população feminina (35,1\%) em comparação aos homens (17,5\%) (Romero 
et al, 2021).

Em um estudo coorte realizado no período de 2020 nos Estados Unidos, foi observado o impacto que a pandemia da Covid-19 teve na saúde mental de idosos. Nesse contexto, foram identificados fatores de riscos específicos para o surgimento de quadros depressivos relacionados ao isolamento social, os quais incluem idade maior ou igual a 70 anos, morar só, ausência de familiares, excesso de álcool, doença crônica, doenças cardíacas, deficiência cognitiva e funcional e pessoas que perderam parentes ou amigos em decorrência da Covid-19 (Briggs, 2021).

É importante que os profissionais de saúde observem o idoso como um todo, em seu aspecto físico, biológico, psicológico e social, pois esta população apresenta mais riscos frente a sintomatologia causada pela doença, sendo de extrema importância esse olhar diferenciado para assegurar a saúde. Visto que com o isolamento social há também os comportamentos depressivos ocasionados pelo próprio isolamento da pandemia, no qual o idoso tem o sentimento de solidão e de prisão (Costa, 2020).

\section{Conclusão}

Atualmente a multimorbidade é uma realidade vivenciada no Brasil pela população idosa, somando-se ao envelhecimento natural ao quadro de doenças crônicas, a infecção aguda causada pelo Coronavírus pode ser muito mais grave nesses pacientes. Segundo os autores, a principal complicação e que frequentemente culmina em óbito é a Síndrome da Angústia Respiratória Aguda. Os quadros mais graves nos idosos estão relacionados a a diminuição do tamanho dos telômeros e no número de linfócitos. Os idosos infectados pelo Sars-Cov-2 podem manifestar a doença tanto de forma assintomática quanto de forma sintomática, as principais repercussões são febre, tosse, falta de ar, fadiga e sintomas gastrointestinais, os indivíduos infectados podem evoluir com pneumonia grave, complicações cardiovasculares, lesão renal, acidente vascular cerebral, gerando morbidade e mortalidade.

É indiscutível que a Covid-19é uma doença severa, que vem causando problemas multifatoriais para a sociedade, dentre esses acometimentos podemos citar os aspectos físicos, emocionais e sociais. Após a análise dos artigos foi percebido que os idosos são os principais acometidos com as repercussões da infecção causada pelo COVID-19. Ao abordarem os aspectos emocionais e sociais como complicações da Covid-19, os autores apontam como principais repercussões o impacto na saúde mental dos idosos impulsionados principalmente pelo isolamento social. Nesse sentido, observou-se o aumento do sentimento de solidão e de tristeza, quadros de depressão e ansiedade, insônia, bem como o comprometimento cognitivo nessa população, todos esses aspectos impulsionam para um risco aumentado ao suicídio. Esses sintomas foram mais presentes no sexo feminino.

Conclui-se então que a doença ocasionada pelo Sars-Cov-2 ainda é recente e algumas das suas repercussões e fisiopatologia ainda são desconhecidas. Além disso, o estudo foi realizado antes do advento da vacina para essa população. Por isso, torna-se necessário outros estudos relacionados a essa temática para melhor compreender aspectos inerentes a infecção por Covid-19 nessa população.

\section{Referências}

Baptista, A. B. \& Fernandes, L. V. V. (2020). COVID-19, análise das estratégias de prevenção, cuidados e complicações sintomáticas. Revista Interdisciplinar da Universidade Federal do Tocantins, 7(3), 38-47.

Batista, S. R., Souza, A. S. S. D., Nogueira, J., Andrade, F. B. D., Thumé, E., Teixeira, D. S. D. C., \& Nunes, B. P. (2020). Comportamentos de proteção contra COVID-19 entre adultos e idosos brasileiros que vivem com multimorbidade: iniciativa ELSI-COVID-19. Cadernos de Saúde Pública, 36, e00196120.

Begam, N. \& Bashar, M. D. Distúrbios olfativos e do paladar em pacientes com infecção por SARS-CoV-2. Arquivos Internacionais de Otorrinolaringologia, $24,391-392$.

Beghi, E., Feigin, V., Caso, V., Santalucia, P. \& Logroscino, G. (2020) Infecção por COVID-19 e complicações neurológicas: resultados atuais e previsões futuras. Neuroepidemiology, 54(4), 1-6. 
Research, Society and Development, v. 11, n. 2, e0811223910, 2022

(CC BY 4.0) | ISSN 2525-3409 | DOI: http://dx.doi.org/10.33448/rsd-v11i2.23910

Briggs, R. McDowell, C. P., Looze, C., Kenny, R. A. \& Ward, M. (2021). Sintomas depressivos entre adultos mais velhos antes e depois da pandemia de COVID19. Journal of the American Medical Directors Association, 22, 2251 a 2257.

Costa, F.A., Silva, A. S., Oliveira, C. B. S., Costa, L. S. C., Paixão, M. E. S., Celestino, M. N. S. \& Santos, I. L. V. L. (2020) COVID-19: seus impactos clínicos e psicológicos na população idosa. Brazilian Journal of Development, 6(7), 49811-49824.

Da Costa R. A. F., Santos, G. S. N. F. \& Monteiro, A. C. C. (2020). Impacto do COVID-19 na qualidade de vida dos idosos. Anais do CIEH.

Galvão, M. H. R. \& Roncalli, A. G. (2020). Fatores associados a maior risco de ocorrência de óbito por COVID-19: análise de sobrevivência com base em casos confirmados. Revista Brasileira de Epidemiologia, 23.

Gerhardt, T. E. \& Silveira, D. T. (2009). Métodos de pesquisa. Porto Alegre: Editora da UFRGS.

He, F., Deng, Y., \& Li, W. (2020) Doença por coronavírus 2019: o que sabemos? Journal of medical virology , 92(7), 719-725.

Mongelli, A., Barbi, V., Zamperla, M. G., Atlante, S., Forleo, L., Nesta, M. \& Gaetano, C. (2021). Evidência para aceleração biológica da idade e encurtamento do telômero em sobreviventes de COVID-19. International Journal of Molecular Sciences, 22(11), 6151.

Moraes, E. N. D., Viana, L. D. G., Resende, L. M. H., Vasconcellos, L. D. S., Moura, A. S., Menezes, A., \& Rabelo, R. (2020). COVID-19 nas instituições de longa permanência para idosos: Estratégias de rastreamento laboratorial e prevenção da propagação da doença. Ciência \& Saúde Coletiva [online] , 25 (9), 3445 3458 .

Nunes, B., Souza, A.S., Nogueira, J., Andrade, F., Thumé, E., Teixeira, D., \& Batista, S. (2020). Envelhecimento, multimorbidade e risco para COVID-19 grave: ELSI-Brasil. Health Sciences.

Portela, V., \& Cebola, M. (2021). Prognóstico da COVID -19 em idosos institucionalizados e com desnutrição, fragilidade e sarcopenia: revisão de escopo. Acta Port Nutr, 24,64-69.

Robinson, L. A. \& Pierce, C. M. Is 'inflamaging' fuelling grave COVID-19 disease? Jornal da Royal Society of Medicine, 113(9), 346-349.

Romero, D. E., Muzy, J., Damacena, G. N., Souza, N. A., Almeida, W. S., Szwarcwald, C. L. \& Silva, D. R. P. (2021) Idosos no contexto da pandemia de COVID-19 no Brasil: reflexos na saúde, renda e trabalho. Cadernos de Saúde Pública, 37.

Saban-Ruiz, J. \& Ly-Pen, D. (2020). COVID-19: uma abordagem cardiometabólica personalizada para reduzir complicações e custos. O papel do envelhecimento para além dos tópicos. $O$ jornal de nutrição, saúde e envelhecimento, 1.

Salimi, S., \& Hamlyn, J. M. (2020). COVID-19 e crosstalk com as marcas do envelhecimento. The Journals of Gerontology: Series A, 75(9), e34-e41.

Sharma, P. \& Sharma, R. (2021). Impact of Covid-19 on mental health and aging. Saudi Journal of Biological Sciences, 28(12), 7946-7053.

Tosato, M., Carfi, A., Marte, I., Pais, C., Ciciarello, F., Rota, E. \& Landi, F. (2021). Prevalência e preditores de persistência dos sintomas de COVID-19 em adultos mais velhos: um estudo de centro único. Journal of the American Medical Directors Association, 22(9), 1840-1844. 